\title{
Healthcare Utilization and Cost Burden of Porphyria in Commercially Insured Adults in the United States
}

\author{
Mohamed I. Elsaid ${ }^{1,2} \cdot$ You $\mathrm{Li}^{1,2} \cdot$ Carolyn Catalano ${ }^{1,2} \cdot$ Carlos D. Minacapelli ${ }^{1,2} \cdot$ Kapil Gupta $^{1,2} \cdot$ Vinod K. Rustgi $^{1,2}$ (1)
}

Published online: 8 September 2020

(c) The Author(s) 2020

\begin{abstract} (US) using real-world claims data. tient visits (US\$12,378 vs US\$9801). admissions, outpatient visits, and pharmaceutical claims.

\section{Introduction}

Porphyrias are a group of rare metabolic disorders caused by dysfunction along the heme biosynthesis pathway [1]. There are eight distinct porphyrias, each caused by enzyme deficiencies that lead to disturbances in the pattern of
\end{abstract}

Objectives The healthcare burden associated with porphyria remains unevaluated despite the associated increased risks of morbidity and mortality. We aimed to assess the healthcare utilization and cost burdens of porphyria in the United States

Methods We performed a case-control analysis of adults in the Truven Health MarketScan ${ }^{\circledR}$ Commercial Claims database (2010-2015). Using propensity scores, 2788 porphyria cases were matched 1:1 to porphyria-free controls with chronic liver disease. Total and service-specific parameters were quantified for the 12 months before porphyria diagnosis versus the 12 months after diagnosis and over the 12 months following a randomly selected date for controls. Wilcoxon signed rank tests and McNemar tests were used to examine incremental differences in burden between cases and controls. Adjusted multivariable generalized linear regression models were used to compare healthcare burdens for cases versus controls.

Results Relative to the 12 months before porphyria diagnosis, the following 12 months had more claims per patient (35.94 vs $39.67 ; p<0.0001$ ) and increased per-patient healthcare costs (US $\$ 21,308$ vs US $\$ 27,270 ; p<0.0001$ ). Porphyria cases incurred US $\$ 7839$ more in total unadjusted costs compared with controls in the 12 months after index date. Compared with controls, cases also had more claims (39.67 vs 34.81), primarily due to inpatient admissions (1.80 vs 0.78 ) and outpatient visits (21.41 vs 17.98). Cases also had higher healthcare costs for inpatient admissions (US\$8882 vs US\$4674) and outpa-

Conclusion Porphyria is associated with significant healthcare costs and utilization burdens driven by increased inpatient

Electronic supplementary material The online version of this article (https://doi.org/10.1007/s41669-020-00229-4) contains supplementary material, which is available to authorized users.

Vinod K. Rustgi

vr262@rwjms.rutgers.edu

Mohamed I. Elsaid

mie10@SPH.Rutgers.edu

You Li

scottlight1186@gmail.com

Carolyn Catalano

ccatalano1025@gmail.com

Carlos D. Minacapelli

minacacd@rwjms.rutgers.edu accumulation and excretion of porphyrin and porphyrin precursors along the pathway [2]. Typically, porphyrias are classified according to the primary site of overproduction of these heme precursors as either hepatic or erythropoietic $[2,3]$ and categorized as either acute or non-acute types [4]. Common clinical signs of porphyria include unexplained abdominal pain and neuropsychiatric symptoms, especially in acute hepatic porphyrias (AHP), while photosensitivity

Kapil Gupta

kg733@rwjms.rutgers.edu

1 Division of Gastroenterology and Hepatology, Department of Medicine, Rutgers Robert Wood Johnson Medical School, 1 Robert Wood Johnson Place, Medical Education Building, Rm \# 466, New Brunswick, NJ 08901, USA

2 Center for Liver Diseases and Liver Masses, Rutgers Robert Wood Johnson Medical School, New Brunswick, NJ, USA 


\section{Key Points for Decision Makers}

While quantifying the disease burden is essential in assessing the cost effectiveness of intervention strategies such as screening, the healthcare burden of porphyria in the United States is unknown.

Our results show that a new porphyria diagnosis results in excess annual healthcare utilization of 3.73 claims per patient and an additional US\$6062 in per-patient healthcare costs. A new diagnosis of porphyria also resulted in an excess per-patient annual cost of US\$7839 when compared with porphyria-free chronic liver disease controls.

Porphyria is associated with significant healthcare cost and utilization burdens driven by increased inpatient admissions and outpatient visits.

is a hallmark of non-acute porphyrias, such as porphyria cutanea tarda (PCT) [4].

In the United States (US), an estimated 200,000 individuals are afflicted by porphyrias [5]. Porphyrias are often misdiagnosed [6] or diagnosed late in the disease process [7]. When appropriately diagnosed, management commonly focuses on reduction of exposure to precipitating factors, such as porphyrinogenic medications, excess alcohol intake, rapid weight loss (fasting), stress, hormonal changes, acute illness/infection, and, in the case of photo-cutaneous porphyrias, exposure to sunlight [8].

European studies have found the prevalence of PCT, the most common porphyria, to be one in 10,000 individuals [5]. While most porphyrias are hereditary, $75-80 \%$ of PCT cases are acquired though exposure to exogenous factors [9] such as alcohol intake, iron overload (hemochromatosis) [10], and chronic hepatitis $\mathrm{C}$ virus (HCV) infection [11-13]. Although multiple forms of porphyria are associated with increased risk of hepatic complications [14, 15], patients with PCT, in particular, are at substantial risk of severe hepatic manifestations [16], including excess mortality from compensated cirrhosis, decompensated cirrhosis (DCC) [17], and hepatocellular carcinoma (HCC) [18]. Evidence exists that individuals with AHP and acute intermittent porphyria (AIP) may also be at greater risk of developing $\operatorname{HCC}[17,19]$. The link between porphyria and HCC is of particular concern given the high prevalence and increasing incidence of HCC, especially among adults aged 60 years and older [20].

Early diagnosis and treatment of porphyria are necessary to prevent increased morbidity and mortality risks due to hepatic complications [14]. Therapeutic phlebotomy or hydroxychloroquine to deplete iron stores is effective in PCT. In addition, eradication of $\mathrm{HCV}$ in all patients with
PCT is recommended [21]. Heme therapy can be provided prophylactically to control symptoms of AHP after hospitalization for an acute attack [22]. Recently, an approach using small interfering RNA was approved by the FDA [23]. Novel therapies that target specific sites of heme overproduction, which could offer promising options for some symptomatic patients, are also being developed [24].

Few studies have evaluated the economic and healthcare burdens of porphyria. A retrospective case-control study conducted in the Netherlands quantified porphyria disease burden in a sample of AIP patients with varying expressions of disease. In this study, data were collected from patient records and questionnaires between 1960 and 2016. Lifetime healthcare cost for patients with recurrent attacks was $€ 5.5$ million greater than for those with symptomatic AIP without recurrent attacks ( $€ 0.3$ million) [25]. These results, however, are limited to one small sample in a single center in the Netherlands, and do not represent the case for the economic and healthcare utilization burdens of porphyria in the US.

Porphyrias are a debilitating set of diseases with significant clinical sequelae. Yet, the economic and healthcare utilization burdens associated with porphyria in the US remain unevaluated. Health insurance claims comprise real-world data that can be used to estimate healthcare costs and utilization for porphyria patients while adjusting for individuals' demographics and disease characteristics. In this study, real-life claims data were used to conduct a propensity score matched case-control analysis to quantify the healthcare resource utilization and economic burdens of porphyria in commercially insured US adults. Evaluation of economic and healthcare burdens can facilitate assessment of intervention strategies, such as determining the cost effectiveness of new therapies and enhancing resource allocation efforts.

\section{Methods}

\subsection{Data Source}

We conducted a case-control study using the Truven Health MarketScan ${ }^{\circledR}$ Commercial Claims (MSCC) databases from January 1, 2010, to December 31, 2015. Data included in the MSCC databases represent national healthcare records from government and public organizations, large employers, and health plans from more than 350 million payers annually. The MSCC databases include longitudinal individual-level data for health insurance claims across inpatient, outpatient, and outpatient prescription drug services. Aggregate data on cost of care included in the MSCC represent the amounts eligible for payment before applying coordination of benefits, deductibles, and copayments. All MSCC records are de-identified data that are compliant with all US patient confidentiality requirements. The Internal Review Board of 
Rutgers Robert Wood Johnson Medical School approved the protocol of this study.

\subsection{Study Sample}

We used inpatient admissions and outpatient records from January 1, 2011 to December 31, 2014 to classify participants as either porphyria patients or chronic liver disease (CLD) patients without porphyria. Porphyria was identified as one primary or secondary record of International Classification of Disease, Ninth Revision (ICD-9) code 277.1 in either inpatient admissions or outpatient services. The CLD group included participants with one primary or secondary ICD-9 code for liver transplant, HCC, DCC, compensated cirrhosis, HCV, chronic hepatitis B (HBV), alcoholic fatty liver, hemochromatosis, non-alcoholic fatty liver disease (NAFLD), autoimmune hepatitis, hepatitis E virus, primary biliary cirrhosis (PBC), or primary sclerosing cholangitis (PSC). Due to small sample size, we combined patients with autoimmune hepatitis, hepatitis E virus, PBC, or PSC into one group (i.e., other chronic liver disease).

An index date was defined for each participant as either the earliest date of porphyria diagnosis, for potential cases, or a randomly selected date from all claim records starting with the earliest record with a CLD diagnosis, for potential porphyria-free controls. The random selection of an index date for controls allowed for the comparison between the burden of porphyria and the average CLD burden across all stages of disease spectrums. We then defined a baseline period for each participant as the 12 months prior to the selected index date, while the study follow-up period represented the 12 months following the defined index date. Only adult $(18+$ years $)$ participants with at least 12 months of enrollment before and after the index date were included in the study.

Baseline demographics, including age, gender, the region of residence, and the type of health insurance plan, were obtained from the index date records. A comorbidity profile was measured for each participant during the baseline period using ICD-9 codes acquired from the inpatient admissions and outpatient services. The profile included participants' statuses on alcohol abuse or dependence (AAD), liver transplant, HCC, DCC, compensated cirrhosis, alcoholic fatty liver, HCV, HBV, hemochromatosis, NAFLD, and other chronic liver disease. In addition, a total weighted Charlson Comorbidity Index (CCI) score was estimated for each participant during the baseline period using algorithms provided by Quan et al. [26].

\subsection{Matching Procedure}

We used propensity score matching to ensure the comparability between cases and controls on all observed baseline demographics and comorbidity profiles. A multivariate logistic regression model, with porphyria status as the outcome, that included age group, gender, the region of residence, the type of health insurance, hemochromatosis, CCI, AAD, liver transplant, HCC, DCC, compensated cirrhosis, alcoholic fatty liver, $\mathrm{HBV}, \mathrm{HCV}$, hemochromatosis, NAFLD, and other CLD, was used to estimate the propensity score for each participant (Supplementary Table S1, see electronic supplementary material [ESM]). In turn, the estimated propensity scores were used to match porphyria cases $1: 1$ to porphyria-free controls using the nearest neighbor matching without replacement strategy (i.e., GREEDY algorithm), while matching exactly on age group and gender [27].

\subsection{Healthcare Utilization and Costs}

The healthcare utilization parameters included the number of claims per patient for inpatient admissions, emergency department (ED) visits, outpatient visits, and pharmaceutical prescriptions. As for the economic burden, we aggregated the total annual service-specific healthcare charges over claims related to inpatient admissions, ED visits, outpatient visits, and pharmaceutical prescriptions. To assess the burden associated with a new porphyria diagnosis, we aggregated the healthcare utilization and cost parameters over the 12 months before and the 12 months after first porphyria diagnosis. In addition, we quantified all utilization and cost parameters over the 12 months following the randomly selected index date for porphyria-free controls to compare the burden of porphyria with that of matched CLD patients without porphyria.

Measures of healthcare utilization included the mean, median, and $25 \mathrm{th} / 75$ th percentiles of the number of claims per patient for inpatient admissions, ED visits, outpatient visits, and pharmaceutical prescriptions. We also estimated the prevalence of having at least one inpatient admission, ED visit, and outpatient visit. An ED visit denoted emergency services that did not result in a hospital admission. The average lengths of inpatient stay per patient for all participants were also calculated for all cases before and after first diagnosis and during the 12 months following index date for controls.

The mean, median, and $25 \mathrm{th} / 75$ th percentiles of the healthcare expenditures were calculated for both the overall and service-specific costs before and after first diagnosis for cases and during the 12 months following the index date for controls. In a sub-analysis, we quantified age-group-specific healthcare cost comparisons between the 12 months before and after first porphyria diagnosis for all five expenditure variables. All costs were adjusted to 2019 United States Dollars (US\$) using the medical care commodities component of the Consumer Price Index [28]. 


\subsection{Statistical Analysis}

We compared baseline characteristics and comorbidity profiles for those with and without porphyria before and after matching using the standardized differences of means and proportions. We used standardized difference cutoffs of 0.2 , 0.5 , and 0.8 to indicate small, medium, and large differences between means and proportions of the two comparison groups (Table 1) [29, 30]. Wald Chi-square tests were performed to test the associations between porphyria status, patients' categorical characteristics, and comorbidity profiles in the unmatched sample. Wilcoxon signed rank tests were used to compare all continuous measures of healthcare costs and utilization.

McNemar tests were used to compare dichotomous parameters of healthcare utilization in both the pre/post-first porphyria diagnosis and in the case versus control analyses.

To quantify the burden of porphyria amongst CLD patients, we conducted secondary multivariable regression analyses using generalized linear models (GLM) models with negative binomial distributions for healthcare utilization rates and gamma distributions for cost estimates. We used generalized estimation equation (GEE) with an exchangeable structure to account for the correlation between cases and controls. All GLM models were adjusted for age group, gender, the region of residence, the type of health insurance, CCI category, AAD, liver transplant, HCC, DCC, compensated cirrhosis, alcoholic fatty liver, HBV, HCV, hemochromatosis, NAFLD, and other CLD. The results of the GLM analyses represented adjusted incidence rate ratios (aIRR) stratified by CLD group for utilization and cost comparisons between cases with porphyria and porphyria-free controls (Supplementary Tables S2-S7, see ESM). In a sensitivity analysis, we restricted our healthcare cost and utilization assessment to cases with at least two records of porphyria diagnosis and controls with at least two records of chronic liver disease diagnosis. $p$ values and 95\% confidence intervals (CIs) were calculated for mean differences and all aIRR estimates. A $p$ value $(p)$ of $<0.05$ was considered statistically significant. All analyses were performed using the SAS 9.4 software (SAS Institute, NC, USA).

\section{Results}

\subsection{Sample Characteristics}

The study sample included 2.04 million participants in MSCC databases who had at least one primary or secondary CLD or porphyria diagnosis in either inpatient admission or outpatient services (Fig. 1). Of all CLD and porphyria patients, 626,604 were adults who met the inclusion criteria of continuous enrollment for 12 months before and after the index date. A total of 2788 porphyria patients who met the inclusion criteria were identified during the study period, all of whom were matched 1:1 using propensity scores to CLD controls. The incidence of porphyria in the unmatched sample was found to be $0.44 \%$.

Between-group differences in the unmatched and matched samples are summarized in Table 1. Compared with those without, porphyria patients were slightly younger (48.28 vs 48.82 years; $p=0.0178$ ) with a higher proportion of females $(55.13 \%$ vs $52.31 \% ; p=0.0029)$ and a lower CCI $(1.10$ vs $1.45 ; p<0.0001)$. Porphyria patients had a lower prevalence of NAFLD ( $4.59 \%$ vs $58.68 \% ; p<0.0001)$, hemochromatosis $(1.83 \%$ vs $6.87 \%$; $p<0.0001)$, HBV $(0.25 \%$ vs $2.96 \%$; $p<0.0001)$, DCC $(1.94 \%$ vs $12.49 ; p<0.0001)$, compensated cirrhosis $(1.22 \%$ vs $23.77 \%$; $p<0.0001), \mathrm{HCC}(0.18 \%$ vs $0.78 \% ; p=0.0003)$, and liver transplant $(0.14 \%$ vs $0.84 \%$; $p<0.0001)$ compared with the porphyria-free CLD cohort. The distribution of all baseline characteristics was balanced between the matched porphyria cases and controls (standardized difference $<0.2$ ) (Table 1).

\subsection{Healthcare Utilization}

Healthcare utilization was compared between the 12-month baseline period (pre-porphyria) and 12 months following the index date (post-porphyria) in the 2788 patients with a record of porphyria (Table 2). The total number of claims per patient was significantly higher in the post-porphyria period than in the pre-porphyria baseline period (pre vs post, 35.94 vs $39.67 ; p<0.0001$ ), representing an increase of 3.73 (95\% CI 2.84-4.62) claims per patient post-diagnosis. The post-porphyria period was characterized by significantly higher numbers of inpatient admissions ( 1.42 vs $1.75 ; p<0.0001)$, outpatient visits (19.36 vs $21.41 ; p<0.0001)$, and pharmaceutical claims $(14.48$ vs $15.84 ; p<0.0001)$ compared with the pre-porphyria period. The prevalence of at least one inpatient admission was significantly higher after the first porphyria diagnosis compared with the pre-porphyria baseline period $(14.38 \%$ vs $17.07 \% ; p=0.0003)$. The average length of inpatient stay for porphyria patients was slightly higher after first diagnosis than in the prediagnosis period, but the difference was not statistically significant ( 1.66 days vs 1.80 days; $p=0.612$ ).

Healthcare utilization was next compared between matched porphyria cases and CLD controls (Supplementary Table S8, see ESM). The total number of claims per patient over the 12 months following index date was significantly higher in the case group than in the control group (case vs control, 39.67 vs $34.81 ; p<0.0001$ ). The increase in the total annual number of claims in porphyria cases compared with controls was related to a higher number 
Table 1 Baseline characteristics of the study sample of patients by porphyria status before and after matching, 2011-2014

\begin{tabular}{|c|c|c|c|c|c|}
\hline Patient characteristics ${ }^{\mathrm{a}}$ & Porphyria $(n=2788)$ & No porphyria $(n=623,816)$ & $\begin{array}{l}\text { Standardized } \\
\text { difference before } \\
\text { matching }^{\mathrm{b}}\end{array}$ & $\begin{array}{l}\text { Matched }^{\mathrm{c}} \text { no } \\
\text { porphyria controls } \\
(n=2788)\end{array}$ & $\begin{array}{l}\text { Standardized } \\
\text { difference after } \\
\text { matching }\end{array}$ \\
\hline Age, mean (SD) & $48.28(11.40)$ & $48.82(10.77)$ & 0.0462 & $48.38(11.18)$ & 0.0088 \\
\hline Age group, $n(\%)$ & & & 0.0663 & & N/A \\
\hline $18-34$ years & $388(13.92)$ & $73,854(11.84)$ & & $388(13.92)$ & \\
\hline 35-44 years & $487(17.47)$ & $117,040(18.76)$ & & $487(17.47)$ & \\
\hline $45-54$ years & $886(31.78)$ & $201,835(32.46)$ & & $886(31.78)$ & \\
\hline $55+$ years & $1027(36.84)$ & $231,087(37.04)$ & & $1027(36.84)$ & \\
\hline Gender, $n(\%)$ & & & 0.0566 & & N/A \\
\hline Male & $1251(44.87)$ & $297,522(47.69)$ & & $1251(44.87)$ & \\
\hline Female & $1537(55.13)$ & $326,294(52.31)$ & & $1537(55.13)$ & \\
\hline $\begin{array}{l}\text { US region of residence, } \\
n(\%)\end{array}$ & & & 0.0474 & & 0.1125 \\
\hline Northeast & $569(20.41)$ & $138,754(22.24)$ & & $672(24.10)$ & \\
\hline North Central & $503(18.04)$ & $111,794(17.92)$ & & $485(17.40)$ & \\
\hline South & $1122(40.24)$ & $244,103(39.13)$ & & $1134(40.67)$ & \\
\hline West & $539(19.33)$ & $118,254(18.96)$ & & $450(16.14)$ & \\
\hline Unknown & $55(1.97)$ & $10,911(1.75)$ & & 47 (1.69) & \\
\hline $\begin{array}{l}\text { Type of health insurance, } \\
n(\%)\end{array}$ & & & 0.0522 & & 0.1597 \\
\hline $\begin{array}{l}\text { Preferred provider } \\
\text { organization }\end{array}$ & $1754(62.91)$ & $381,649(61.18)$ & & $1540(55.24)$ & \\
\hline $\begin{array}{l}\text { Health maintenance } \\
\text { organization }\end{array}$ & $314(11.26)$ & $79,954(12.82)$ & & $394(14.13)$ & \\
\hline Comprehensive & $85(3.05)$ & $17,670(2.83)$ & & $110(3.95)$ & \\
\hline Point of service & $219(7.86)$ & $50,270(8.06)$ & & $271(9.72)$ & \\
\hline Other & $416(14.92)$ & $94,273(15.11)$ & & $473(16.97)$ & \\
\hline \multicolumn{6}{|l|}{ Comorbidity profile $^{\mathrm{d}}$} \\
\hline $\begin{array}{l}\text { Charlson comorbidity } \\
\text { index, mean (SD) }\end{array}$ & $1.10(1.77)$ & $1.45(2.09)$ & 0.1821 & $1.18(1.81)$ & 0.0467 \\
\hline $\begin{array}{l}\text { Charlson comorbidity } \\
\text { index, } n(\%)\end{array}$ & & & 0.1967 & & 0.0599 \\
\hline 0 & $1440(51.65)$ & $271,497(43.52)$ & & $1379(49.46)$ & \\
\hline 1 & $661(23.71)$ & $153,450(24.60)$ & & $655(23.49)$ & \\
\hline 2 & $283(10.15)$ & $73,971(11.86)$ & & $317(11.37)$ & \\
\hline 3 & $193(6.92)$ & $50,345(8.07)$ & & $199(7.14)$ & \\
\hline $4+$ & $211(7.57)$ & $74,553(11.95)$ & & $238(8.54)$ & \\
\hline $\begin{array}{l}\text { Alcohol abuse or depend- } \\
\text { ence, } n(\%)\end{array}$ & $22(0.79)$ & $11,311(1.81)$ & 0.0905 & $27(0.97)$ & 0.0192 \\
\hline Liver transplant, $n(\%)$ & $4(0.14)$ & $5239(0.84)$ & 0.0997 & $4(0.14)$ & N/A \\
\hline $\begin{array}{l}\text { Hepatocellular carcinoma, } \\
n(\%)\end{array}$ & $5(0.18)$ & $4846(0.78)$ & 0.0867 & $4(0.14)$ & 0.0894 \\
\hline $\begin{array}{l}\text { Decompensated cirrhosis, } \\
n(\%)\end{array}$ & $54(1.94)$ & 77,927 (12.49) & 0.4167 & $54(1.94)$ & N/A \\
\hline $\begin{array}{l}\text { Compensated cirrhosis, } \\
n(\%)\end{array}$ & $34(1.22)$ & $23,542(3.77)$ & 0.1643 & $33(1.18)$ & 0.0329 \\
\hline Alcoholic fatty liver, $n(\%)$ & $9(0.32)$ & $11,061(1.77)$ & 0.1428 & $9(0.32)$ & N/A \\
\hline Hepatitis $\mathrm{C}$ virus, $n(\%)$ & $116(4.16)$ & $60,019(9.62)$ & 0.2168 & $147(5.27)$ & 0.5247 \\
\hline Hepatitis B virus, $n(\%)$ & $7(0.25)$ & $18,464(2.96)$ & 0.2168 & $7(0.25)$ & N/A \\
\hline Hemochromatosis, $n(\%)$ & $51(1.83)$ & $42,886(6.87)$ & 0.2492 & $52(1.87)$ & 0.0027 \\
\hline $\begin{array}{l}\text { Non-alcoholic fatty liver, } \\
n(\%)\end{array}$ & $128(4.59)$ & $366,071(58.68)$ & 1.4298 & $128(4.59)$ & N/A \\
\hline
\end{tabular}


Table 1 (continued)

\begin{tabular}{|c|c|c|c|c|c|}
\hline Patient characteristics ${ }^{\mathrm{a}}$ & Porphyria $(n=2788)$ & No porphyria $(n=623,816)$ & $\begin{array}{l}\text { Standardized } \\
\text { difference before } \\
\text { matching }^{\mathrm{b}}\end{array}$ & $\begin{array}{l}\text { Matched }^{\mathrm{c}} \text { no } \\
\text { porphyria controls } \\
(n=2788)\end{array}$ & $\begin{array}{l}\text { Standardized } \\
\text { difference after } \\
\text { matching }^{\mathrm{b}}\end{array}$ \\
\hline $\begin{array}{l}\text { Other chronic liver } \\
\text { diseases }{ }^{\mathrm{e}}, n(\%)\end{array}$ & $5(0.18)$ & $11,294(1.81)$ & 0.1649 & $5(0.18)$ & N/A \\
\hline
\end{tabular}

N/A exactly matched or identical number of cases and controls, $S D$ standard deviation

${ }^{a}$ All demographics data were obtained on index date (first diagnosis date of porphyria for cases and a randomly selected date from all claim records for controls) for chronic liver disease adult participants with continuous enrollment during the 12 months prior and the 12 months following index date

${ }^{b}$ Difference in means or proportions divided by the standard error. Smaller values indicate better balance between cases and controls

${ }^{c}$ Porphyria cases and porphyria-free controls with chronic liver disease were matched 1:1 using propensity score. The logistic regression model used to estimate the propensity scores included age group, region of residence, gender, type of health insurance, Charlson comorbidity index, alcohol abuse or dependence liver transplant, hepatocellular carcinoma, decompensated cirrhosis, compensated cirrhosis, alcoholic fatty liver, hepatitis $\mathrm{C}$ virus, hepatitis B virus, hemochromatosis, non-alcoholic fatty liver, and other chronic liver disease. In turn, cases and controls were exactly matched on age group and gender

${ }^{\mathrm{d}}$ Was estimated during the 12 months prior to the index date

${ }^{\mathrm{e}}$ Included patients with autoimmune hepatitis, hepatitis E virus, primary biliary cirrhosis, or primary sclerosing cholangitis

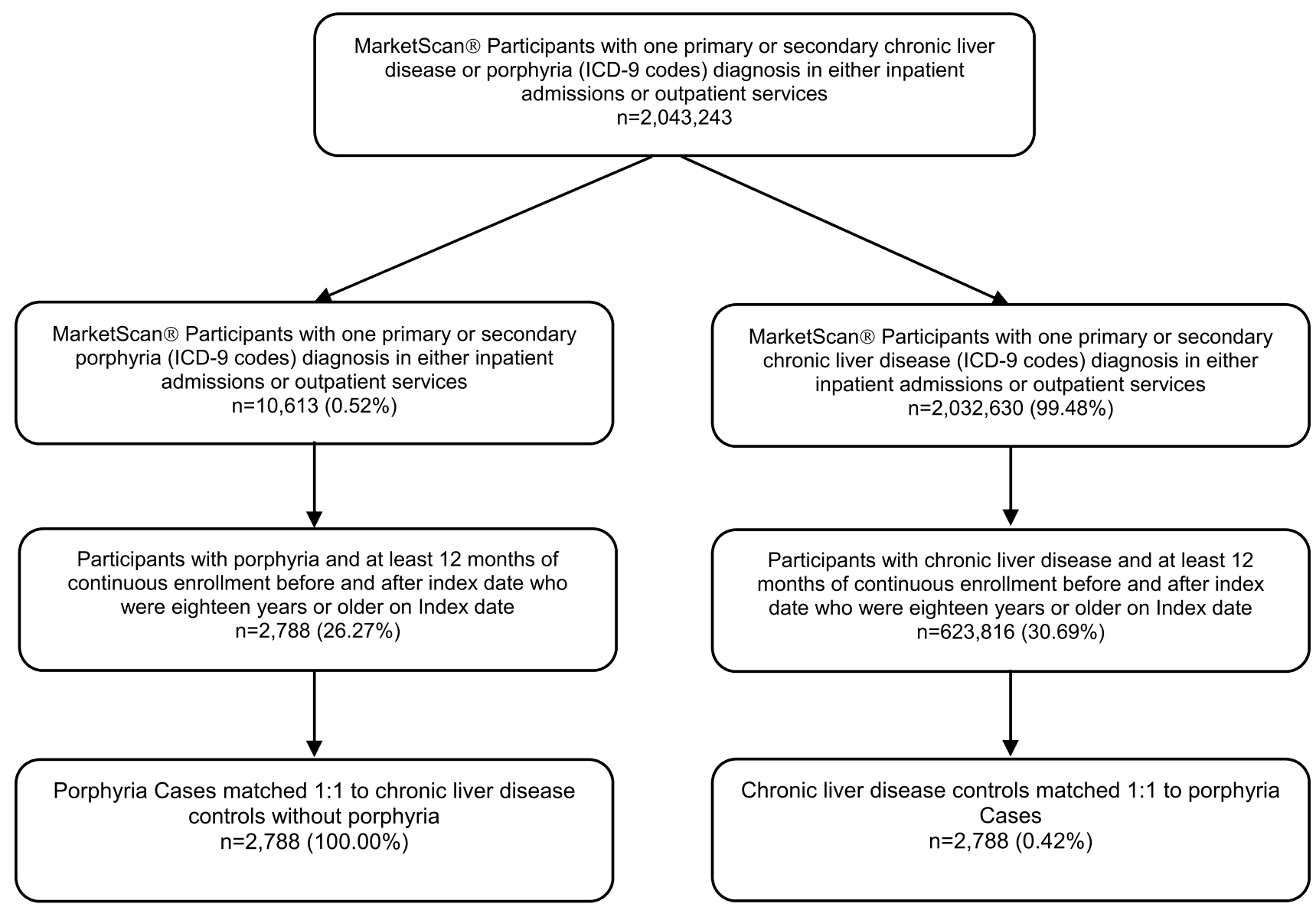

Fig. 1 Diagram for study sample selection

of inpatient admissions (case vs control, 1.75 vs 0.87 ; $p<0.0001$ ) and outpatient visits (case vs control, $21.41 \mathrm{vs}$ $17.98 ; p<0.0001)$. The prevalence of at least one inpatient admission was significantly higher for porphyria cases compared with controls $(17.07 \%$ vs $11.66 \%$; $p<0.0001)$. We also observed a significant higher average length of inpatient 
Table 2 Healthcare resource utilization for porphyria patients during the 12 months before versus the 12 months after first diagnosis date, 2011-2014

\begin{tabular}{|c|c|c|c|}
\hline Healthcare utilization & Pre-diagnosis $(n=2788)$ & Post-diagnosis $(n=2788)$ & $p$ value* \\
\hline \multicolumn{4}{|l|}{ Total number of claims } \\
\hline Mean (SD) & $35.94(33.74)$ & $39.67(35.92)$ & \multirow[t]{2}{*}{$<0.0001$} \\
\hline Median [25th, 75th percentile] & $26[13,49]$ & $30[15,52.5]$ & \\
\hline \multicolumn{4}{|l|}{ Inpatient admissions } \\
\hline Prevalence of at least one visit, $n(\%)$ & $401(14.38)$ & $476(17.07)$ & 0.0003 \\
\hline \multicolumn{4}{|l|}{ Number of admissions } \\
\hline Mean (SD) & $1.42(8.17)$ & $1.75(8.44)$ & \multirow[t]{2}{*}{$<0.0001$} \\
\hline Median [25th, 75th percentile] & $0[0,0]$ & $0[0,0]$ & \\
\hline \multicolumn{4}{|l|}{ Total length of stay, days } \\
\hline Mean (SD) & $1.66(10.50)$ & $1.80(10.07)$ & \multirow[t]{2}{*}{0.0612} \\
\hline Median [25th, 75th percentile] & $0[0,0]$ & $0[0,0]$ & \\
\hline \multicolumn{4}{|l|}{ Emergency department visits } \\
\hline Prevalence of at least one visit, $n(\%)$ & $741(26.58)$ & $693(24.86)$ & 0.0752 \\
\hline \multicolumn{4}{|l|}{ Number of visits } \\
\hline Mean (SD) & $0.68(2.58)$ & $0.67(2.61)$ & \multirow[t]{2}{*}{0.1752} \\
\hline Median [25th, 75th percentile] & $0[0,1]$ & $0[0,0]$ & \\
\hline \multicolumn{4}{|l|}{ Outpatient visits } \\
\hline Prevalence of at least one visit, n (\%) & 2785 (99.89) & $2786(99.93)$ & 0.6547 \\
\hline \multicolumn{4}{|l|}{ Number of visits } \\
\hline Mean (SD) & $19.36(19.84)$ & $21.41(20.95)$ & \multirow[t]{2}{*}{$<0.0001$} \\
\hline Median [25th, 75th percentile] & $13[7,25]$ & $15[8,28]$ & \\
\hline \multicolumn{4}{|l|}{ Pharmaceutical claims } \\
\hline \multicolumn{4}{|l|}{ Number of claims } \\
\hline Mean (SD) & $14.48(16.73)$ & $15.84(17.38)$ & \multirow[t]{2}{*}{$<0.0001$} \\
\hline Median [25th, 75th percentile] & $10[1,22]$ & $11[2,23]$ & \\
\hline
\end{tabular}

N/A not applicable

*For the comparisons between pre- and post-first porphyria diagnosis, all $p$ values were obtained from Wilcoxon signed rank tests for continuous variables and McNemar tests for binary variables stay between cases and controls (1.80 days vs 0.78 days; $p<0.0001)$.

\subsection{Healthcare Costs}

In the pre- versus post-diagnosis analysis, the per-patient total costs of healthcare services were US $\$ 21,308$ and US $\$ 27,370$ for pre-porphyria and post-porphyria, respectively (Table 3). The difference in the total unadjusted cost was significant with post-diagnosis cases costing US $\$ 6062$ (95\% CI 3686-8439) more than pre-diagnosis cases. The incremental difference in annual healthcare cost after versus before first porphyria diagnosis was related to US $\$ 1482$ (24.45\%) in inpatient costs, US\$26 (0.43\%) from ED costs, US\$2919 (48.15\%) from the costs of outpatient visits, and US\$1635 (26.97\%) from outpatient prescription costs.

Healthcare costs were then compared between matched porphyria cases and CLD controls without porphyria (Table 4). The total costs of healthcare services over the 12 months following index dates were significantly higher in porphyria cases than in controls (case vs control,
US $\$ 27,370$ vs US $\$ 19,531 ; p<0.0001)$, an excess perpatient annual cost of US\$7839 (95\% CI 4826-10,852). Incremental annual cost in porphyria compared with controls was related to higher expenditures for inpatient admissions (US\$8882 vs US\$4674; $p<0.0001$ ) and outpatient visits (US\$12,378 vs US $\$ 9801 ; p<0.0001$ ).

The differences in total annual healthcare costs for porphyria patients between pre-diagnosis and post-diagnosis were significant among all age groups except for those 18-34 years old (Fig. 2). The highest difference of US $\$ 7610$ (95\% CI 3888-11,333) in annual total cost between prediagnosis and post-diagnosis was observed in patients aged 45-54 years. Relative to pre-diagnosis, the cost of inpatient services post-diagnosis was significantly higher in ages $45-54$ years (US $\$ 5372$ vs US $\$ 7696 ; p=0.0031$ ) and $55+$ years (US\$5963 vs US $\$ 7840 ; p=0.027$ ). The annual costs related to outpatient visits were significantly higher for patients post-diagnosis in ages 35-44 years (US\$8277 vs US $\$ 10,334 ; p=0.0164$ ), 45-54 years (US\$8949 vs US $\$ 11,939 ; p<0.0001$ ), and $55+$ years (US\$10,231 vs US $\$ 12,936 ; p=0.0003$ ) compared with pre-diagnosis. 
Table 3 Annual per-person all-cause healthcare cost ${ }^{\mathrm{a}}$ comparisons for porphyria patients during the 12 months before versus the 12 months after first diagnosis date, 2011-2014
Table 4 Annual per-person all-cause healthcare cost ${ }^{\mathrm{a}}$ comparisons between porphyria cases and matched chronic liver disease controls without porphyria, 2011-2014

\begin{tabular}{llll}
\hline Healthcare cost ${ }^{\mathrm{a}}(\$ \mathrm{US})$ & Pre-diagnosis $(n=2788)$ & Post-diagnosis $(n=2788)$ & $p$ value $^{\mathrm{b}}$ \\
\hline Total cost & & & \\
Mean (SD) & $21,308(52,775)$ & $27,370(70,674)$ & $<0.0001$ \\
Median [25th, 75th percentile] & $6497(2360,18,298)$ & $7842(3041,21,997)$ & \\
Inpatient admissions & & & 0.0020 \\
Mean (SD) & $7400(39,244)$ & $8882(43,876)$ & \\
Median [25th, 75th percentile] & $0(0,0)$ & $0(0,0)$ & 0.8863 \\
Emergency department visits & & & \\
Mean (SD) & $1370(7082)$ & $0(0,0)$ & $<0.0001$ \\
Median [25th, 75th percentile] & $0(0,263)$ & & $12,378(41,774)$ \\
Outpatient visits & & $4241(1662,10,581)$ & \\
Mean (SD) & $9459(22,834)$ & & \\
Median [25th, 75th percentile] & $3740(1390,9522)$ & $4714(15,672)$ & \\
Pharmaceutical claims & & $718(24,3153)$ & \\
Mean (SD) & $3079(8728)$ & & \\
Median [25th, 75th percentile] & $559(11,2742)$ & & \\
\hline
\end{tabular}

$S D$ standard deviation

${ }^{\text {a }}$ All costs were adjusted to the 2019 United States Dollar (US\$) using the medical care component of the Consumer Price Index

${ }^{\mathrm{b}}$ Wilcoxon signed rank test for cost differences before and after first porphyria diagnosis

\begin{tabular}{llll}
\hline Healthcare cost ${ }^{\mathrm{a}}(\mathrm{US} \$)$ & Porphyria $(n=2788)$ & No porphyria $(n=2788)$ & $p$ value $^{\mathrm{b}}$ \\
\hline Total cost of claims & & & $<0.0001$ \\
Mean (SD) & $27,370(70,674)$ & $19,531(46,287)$ & \\
Median [25th, 75th percentile] & $7842(3041,21,997)$ & $6765(2188,17,569)$ & $<0.0001$ \\
Inpatient admissions & & & \\
Mean (SD) & $8882(43,876)$ & $4674(28,461)$ & 0.0697 \\
Median [25th, 75th percentile] & $0(0,0)$ & $0(0,0)$ & \\
Emergency department visits & & & \\
Mean (SD) & $1396(5950)$ & $0(0,562)$ & \\
Median [25th, 75th percentile] & $0(0,0)$ & & \\
Outpatient visits & & $9801(27,039)$ & \\
Mean (SD) & $12,378(41,774)$ & $3284(1146,8643)$ & \\
Median [25th, 75th percentile] & $4241(1662,10,581)$ & & \\
Pharmaceutical claims & & $3762(11,386)$ & \\
Mean (SD) & $4714(15,672)$ & $553(21,2902)$ & \\
Median [25th, 75th percentile] & $718(24,3153)$ &
\end{tabular}

$S D$ standard deviation

${ }^{a}$ All costs were adjusted to the 2019 United States Dollar (US\$) using the medical care component of the Consumer Price Index. Costs were calculated for the 12 months after first diagnosis of porphyria for cases and for the 12 months following a randomly selected index date for porphyria-free controls

${ }^{\mathrm{b}}$ Wilcoxon signed rank test for cost difference between porphyria cases and porphyria-free controls
Post-diagnosis cases aged 18-34, 35-44, 45-54, and $55+$ years incurred US\$771 (95\% CI 138-1404), US\$902 (95\% CI 118-1685), US\$1982 (95\% CI 966-2998), and US\$2010 (95\% CI 1035-2984) higher pharmaceutical costs compared with pre-diagnosis, respectively (data not shown).

\subsection{Secondary Analysis}

In patients with NAFLD and DCC, porphyria cases had aIRR 1.41 (95\% CI 1.15-1.72) and aIRR 2.19 (95\% CI 1.66-2.90) times the total numbers of claims per patient 


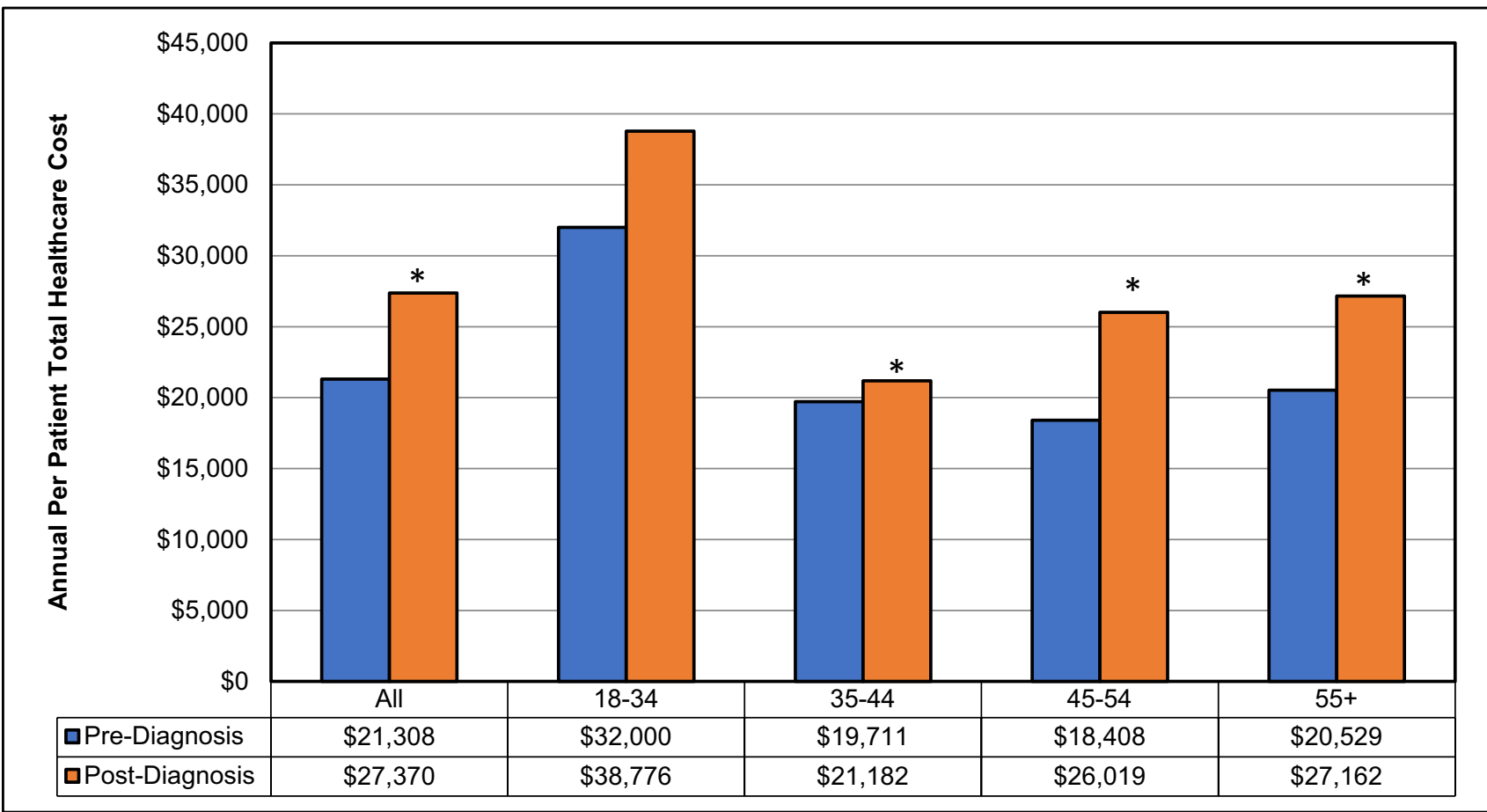

* Wilcoxon Signed Rank Tests P-value $<0.05$ for the total difference in annual per person healthcare cost pre- versus post- First porphyria diagnosis in patients with the same age group.

$\dagger$ All costs were adjusted to 2019 United States Dollar (\$) Using the Medical Care Component of the Consumer Price Index

Fig. 2 Annual per-patient total healthcare $\operatorname{costs}^{\dagger}$ for porphyria patients pre- versus post-first diagnosis by age group (years). *Wilcoxon signed rank tests $p$ value $<0.05$ for the total difference in annual per-person healthcare cost pre- versus post-first porphyria

compared with controls in the 12 months following the index date. Similarly, among NAFLD and DCC patients, the adjusted cost in the 12 months following index date for porphyria cases was aIRR 1.91 (95\% CI 1.36-2.69) and aIRR 4.40 (95\% CI 2.92-6.65), respectively, times the cost for controls. Among patients with alcoholic fatty liver disease and those with liver transplant, the total adjusted annual cost for porphyria cases was aIRR 4.99 (95\% CI 1.56-15.93) and aIRR 2.61 (95\% CI 1.36-5.03), respectively, times the cost for controls with alcoholic liver disease and liver transplant.

\subsection{Sensitivity Analysis}

The results of the sensitivity analysis were consistent with the main findings of significantly higher cost and utilization burdens in porphyria cases versus CLD controls without porphyria. The total number of claims per patient over the 12 months following index date was significantly higher in the case group than in the control group (case vs control, 44.62 vs $37.48 ; p<0.0001)$. The increase in the total annual number of claims in porphyria cases compared with controls was related to a higher number of inpatient admissions and outpatient visits. Similarly, the total costs of healthcare diagnosis in patients within the same age group. ${ }^{\dagger}$ All costs were adjusted to 2019 United States Dollars (\$) Using the Medical Care Component of the Consumer Price Index

services over the 12 months following index dates were significantly higher in porphyria cases than in controls (case vs control, US $\$ 35,604$ vs US $\$ 25,577 ; p<0.0001$ ), an excess per patient annual cost of US\$10,026 (95\% CI 4021-16,031).

\section{Discussion}

To our knowledge, this is the first study to utilize real-life claims data to estimate the healthcare utilization and cost burdens related to porphyria diagnosis in commercially insured US adults. Our case-control analysis highlights the higher cost burden and healthcare utilization by patients diagnosed with porphyria. Findings show that a new porphyria diagnosis results in excess annual healthcare utilization of 3.73 claims per patient and an additional US\$6062 in per-patient healthcare costs. A new diagnosis of porphyria also resulted in an excess per-patient annual cost of US\$7839 when compared with porphyria-free CLD controls.

In our study, increases in the incremental difference in annual per-person healthcare costs post-versus pre-first porphyria diagnosis were primarily due to inpatient costs, 
outpatient visits, and prescription costs. These results may reflect increased costs attributable to initial porphyria diagnosis and evaluation. Similar results were demonstrated in a study of hemochromatosis where a new diagnosis was associated with excess outpatient visits and prescription costs, potentially attributable to the need for phlebotomy sessions [31]. Phlebotomy sessions are accepted first-line therapy for PCT [14]. Outpatient phlebotomy sessions can cost US\$400-US\$1400 per visit [32]. As PCT is the most common porphyria, this would be in line with a post-diagnosis increase in outpatient costs. Other therapies depend on the type of porphyria but include adequate carbohydrate intake and avoiding triggers such as alcohol or certain hormones. Givosiran, a double-stranded small interfering RNA therapeutic targeting delta-aminolevulinic acid synthase 1 , was approved in 2019 for treating AIP [23, 33]. At the time of approval, givosiran had a suggested net price of US $\$ 442,000$ after discounts [34].

The prevalence of at least one inpatient admission was significantly higher after the first porphyria diagnosis compared with the pre-porphyria baseline period. Except for pharmaceutical claims, similar results of increased utilization burden in porphyria were obtained in the case versus control analysis. These findings may be explained by the fact that once a diagnosis of porphyria is established, hospitalization is indicated, especially for acute porphyric attacks, to prevent deterioration of patient status and to permit intravenous administration of hemin or glucose [35, 36].

Total annual healthcare costs related to a new porphyria diagnosis were significantly higher in all age groups, except the youngest (18-34 years old). The highest difference of US $\$ 7610$ in annual total cost between pre-diagnosis and post-diagnosis was found in patients aged 45-54 years. Costs for outpatient visits were also significantly higher for those $35+$ years old and the costs of inpatient admission were higher in ages $45+$ years. Higher pharmaceutical costs existed for post-diagnosis compared with pre-diagnosis in all age groups.

When left untreated, some forms of porphyria can result in long-term sequelae, including severe hepatic manifestations such as HCC [14]. HCC incidence increases with age and amelioration of HCC risk factors, such as eradication of $\mathrm{HCV}$ infection, has less of an effect on hepatocarcinogenesis in older patients [37]. Due to its association with other chronic illnesses, net cost of care for HCC is higher than for other cancers [38]. The significant increase in cost of inpatient admissions in those aged $45+$ years suggests that the higher burden we found in older age cohorts may result from delayed diagnoses, with elevated costs associated with disease progression.

In patients with NAFLD, porphyria cases in our study had 1.41 times the total number of claims and 1.91 times the total adjusted annual cost compared with controls in the
12 months following the index date. Ergen et al. suggest that NAFLD, specifically nonalcoholic steatohepatitis (NASH), is a predisposing factor in triggering porphyria [39]. This is of particular importance given that NAFLD is one of the leading causes of liver disease in the US, and continues to rise in prevalence [40].

Alcohol is a known porphyrinogenic agent and even relatively modest consumption of alcohol $(>60 \mathrm{~g})$ can result in an acute attack [41]. In general, alcohol use has been associated with high healthcare utilization costs and hepatic disease [42]. Alcohol-related liver disease, in particular, is responsible for disproportionate healthcare and cost burdens. In one study, alcoholic cirrhosis comprised over half of total direct healthcare costs for cirrhosis, though it represented only $36 \%$ of all cirrhosis cases. The same study found that in patients with cirrhosis, post-diagnosis per-person costs were nearly twice as much for those with alcoholic cirrhosis versus non-alcoholic cirrhosis (US\$44,835 vs US\$23,319) [43]. This correlates with our finding of higher costs amongst patients with alcoholic fatty liver, where the total adjusted annual cost for porphyria cases were 4.99 times the cost for controls.

Porphyria cases with cirrhosis, both compensated and decompensated, had significantly higher healthcare burdens relative to patients with only DCC. Higher numbers of claims and increased costs for porphyria cases in cirrhosis patients may be explained by increased disease severity among porphyria patients. A cohort study in Denmark found that patients with PCT had more comorbidities and higher risk of mortality than controls [44]. In a large observational study of acute porphyria in the US, patients reported receiving a diagnosis an average of 15 years after experiencing initial symptoms [7]. Due to the potential severity and irreversibility of damage that can be caused by porphyria, management of comorbid conditions, avoidance of triggers, and timely provision of appropriate therapeutics are key components of disease management. This, however, necessitates enhancing provider recognition of the condition to allow early identification of porphyria [8]. Furthermore, although treatment for most porphyrias has not always been optimal [24], early intervention with new therapeutic approaches (such as those targeting specific disturbances in the heme biosynthesis pathway) may prevent the cascade of debilitating disease that can often be associated with this condition.

Our study is not without limitations. The MSCC database is based on ICD-9 codes, which might underestimate the prevalence of some comorbid conditions. Furthermore, the disease diagnostic codes do not contain information on disease severity. Coding errors during the time of claims data entry may lead to misclassifications. However, our findings were confirmed by the sensitivity analysis results in which we limited our sample to cases and controls with at least two confirmed records. The findings of this study may 
not represent the US population, since the MSCC database only covers commercially insured subjects. Our analysis was based on claims data from 2010-2015. However, clinical practices related to porphyria and chronic liver disease have not significantly changed during recent years; hence, use of more recent data is unlikely to alter our main findings. In order to quantify the annual healthcare of porphyria we limited our analysis to patients with 12 months of continuous enrolment before and after porphyria diagnosis. Hence, our results might not represent patients who disenrolled or died within the first year after porphyria diagnosis.

The study has several strengths. This is the first study in the US to utilize real-world claims data to quantify healthcare resource utilization and economic burdens of porphyria. The analysis included a large sample size $(N=617,896)$ and a matched control group to allow for the estimation of incremental differences in healthcare utilization and cost parameters. The use of propensity score matching ensured high comparability between cases and controls. Finally, multiple analyses (pre-first diagnosis vs post-first diagnosis and cases vs controls) ensured accurate estimates of attributable utilization and cost burdens.

\section{Conclusions}

This is the first comprehensive cohort analysis estimating the healthcare cost and burden of porphyria. Our retrospective, population-based claims analysis suggests that porphyria imposes substantially higher healthcare cost and utilization burdens compared with matched controls within a year of disease diagnosis. The burden is mostly due to increased inpatient admissions, outpatient visits, and pharmaceutical claims. Additional research is needed to identify the cost burden of the various types of porphyria to assist providers and payors in further identifying risk based on the specific genotypic/phenotypic expressions of the disease.

Acknowledgements The Rutgers University Institute for Health, Health Care Policy and Aging Research housed the datasets analyzed in this study. Writing assistance: Vital Statistics Consulting helped in formulating a first draft of this manuscript.

Author Contributions MIE designed the analysis; MIE and YL analyzed the data; MIE, CDM, CC and KG wrote the manuscript. CDM provided regulatory support and overall coordination of research required activities. KG and YL provided data support/data collection. MIE, CDM, YL, CC, KG and VKR provided critical editorial comments. VKR provided study concept and was the overall study supervisor. All authors read and approved the final manuscript.

\section{Declarations}

Funding/support This study has received no funding.
Ethics approval The study was reviewed and approved by our Institutional Reviewer.

Consent to participate Not applicable.

Consent for publication The study was reviewed and approved for publication by our Institutional Reviewer.

Availability of data and material The original anonymous dataset is available on request from the corresponding author at vinod.rustgi@ rutgers.edu.

Code availability Software application or custom code.

Financial disclosure The authors in this manuscript have no relevant financial or other relationships to disclose.

Data availability statement All data used in this study were obtained from IBM Watson Health via a restricted access agreement with Rutgers University. As such, the authors cannot make any of the data used in this research publicly available.

Open Access This article is licensed under a Creative Commons Attribution-NonCommercial 4.0 International License, which permits any non-commercial use, sharing, adaptation, distribution and reproduction in any medium or format, as long as you give appropriate credit to the original author(s) and the source, provide a link to the Creative Commons licence, and indicate if changes were made. The images or other third party material in this article are included in the article's Creative Commons licence, unless indicated otherwise in a credit line to the material. If material is not included in the article's Creative Commons licence and your intended use is not permitted by statutory regulation or exceeds the permitted use, you will need to obtain permission directly from the copyright holder. To view a copy of this licence, visit http://creativecommons.org/licenses/by-nc/4.0/.

\section{References}

1. Rigor J, Pinto SA, Martins-Mendes D. Porphyrias: a clinically based approach. Eur J Intern Med. 2019;67:24-9.

2. Balwani M, Desnick RJ. The porphyrias: advances in diagnosis and treatment. Hematol Am Soc Hematol Educ Progr. 2012;2012:19-27.

3. Puy H, Gouya L, Deybach JC. Porphyrias. Lancet. 2010;375(9718):924-37.

4. Stolzel U, Doss MO, Schuppan D. Clinical guide and update on porphyrias. Gastroenterology. 2019;157(2):365.e4-81.e4.

5. Ramanujam VM, Anderson KE. Porphyria diagnostics-part 1: a brief overview of the porphyrias. Curr Protoc Hum Genet. 2015;86:17-20 (1-6).

6. Hudgins K. Porphyria: a rare, complicated, and misdiagnosed disease. Crit Care Nurs Q. 2019;42(2):192-7.

7. O'Malley R, Rao G, Stein P, Bandmann O. Porphyria: often discussed but too often missed. Pract Neurol. 2018;18(5):352-8.

8. Wang B, Rudnick S, Cengia B, Bonkovsky HL. Acute hepatic porphyrias: review and recent progress. Hepatol Commun. 2019;3(2):193-206

9. Ryan Caballes F, Sendi H, Bonkovsky HL. Hepatitis C, porphyria cutanea tarda and liver iron: an update. Liver Int. 2012;32(6):880-93. 
10. Ishak KG, Zimmerman HJ, Ray MB. Alcoholic liver disease: pathologic, pathogenetic and clinical aspects. Alcohol Clin Exp Res. 1991;15(1):45-66.

11. Navas S, Bosch O, Castillo I, Marriott E, Carreno V. Porphyria cutanea tarda and hepatitis $\mathrm{C}$ and $\mathrm{B}$ viruses infection: a retrospective study. Hepatology (Baltimore, MD). 1995;21(2):279-84.

12. Negro F, Forton D, Craxi A, Sulkowski MS, Feld JJ, Manns MP. Extrahepatic morbidity and mortality of chronic hepatitis C. Gastroenterology. 2015;149(6):1345-60.

13. Younossi Z, Park H, Henry L, Adeyemi A, Stepanova M. Extrahepatic manifestations of hepatitis C: a meta-analysis of prevalence, quality of life, and economic burden. Gastroenterology. 2016;150(7):1599-608.

14. Karim Z, Lyoumi S, Nicolas G, Deybach JC, Gouya L, Puy H. Porphyrias: a 2015 update. Clin Res Hepatol Gastroenterol. 2015;39(4):412-25.

15. Anstey AV, Hift RJ. Liver disease in erythropoietic protoporphyria: insights and implications for management. Postgrad Med J. 2007;83(986):739-48.

16. Gumber SC, Chopra S. Hepatitis C: a multifaceted disease Review of extrahepatic manifestations. Ann Intern Med. 1995;123(8):615-20.

17. Linet MS, Gridley G, Nyren O, Mellemkjaer L, Olsen JH, Keehn $\mathrm{S}$, et al. Primary liver cancer, other malignancies, and mortality risks following porphyria: a cohort study in Denmark and Sweden. Am J Epidemiol. 1999;149(11):1010-5.

18. Fracanzani AL, Taioli E, Sampietro M, Fatta E, Bertelli C, Fiorelli $\mathrm{G}$, et al. Liver cancer risk is increased in patients with porphyria cutanea tarda in comparison to matched control patients with chronic liver disease. J Hepatol. 2001;35(4):498-503.

19. Andant C, Puy H, Bogard C, Faivre J, Soule JC, Nordmann Y, et al. Hepatocellular carcinoma in patients with acute hepatic porphyria: frequency of occurrence and related factors. J Hepatol. 2000;32(6):933-9.

20. Rich NE, Yopp AC, Singal AG, Murphy CC. Hepatocellular carcinoma incidence is decreasing among younger adults in the United States. Clin Gastroenterol Hepatol. 2020;18(1):242-248.e5. https ://doi.org/10.1016/j.cgh.2019.04.043.

21. Singal AK. Porphyria cutanea tarda: recent update. Mol Genet Metab. 2019;128(3):271-81. https://doi.org/10.1016/j.ymgme .2019.01.004

22. Thadani H, Deacon A, Peters T. Diagnosis and management of porphyria. BMJ. 2000;320(7250):1647-51.

23. Scott LJ. Givosiran: first approval. Drugs. 2020;80(3):335-9.

24. Fontanellas A, Ávila MA, Anderson KE, Deybach J-C. Current and innovative emerging therapies for porphyrias with hepatic involvement. J Hepatol. 2019;71(2):422-33. https://doi. org/10.1016/j.jhep.2019.05.003

25. Neeleman RA, Wagenmakers M, Koole-Lesuis RH, Mijnhout GS, Wilson JHP, Friesema ECH, et al. Medical and financial burden of acute intermittent porphyria. J Inherit Metab Dis. 2018;41(5):809-17.

26. Quan H, Sundararajan V, Halfon P, Fong A, Burnand B, Luthi JC, et al. Coding algorithms for defining comorbidities in ICD-9-CM and ICD-10 administrative data. Med Care. 2005;43(11):1130-9.

27. Austin PC. A comparison of 12 algorithms for matching on the propensity score. Stat Med. 2014;33(6):1057-69.

28. Statistics; USBoL. Consumer Price Index. 2019 [cited 2019 07/21/2019]; https://www.bls.gov/cpi/. Accessed 3 July 2019
29. Austin PC. Balance diagnostics for comparing the distribution of baseline covariates between treatment groups in propensity-score matched samples. Stat Med. 2009;28(25):3083-107.

30. Cohn J. Statistical power analysis for the behavioral sciences Technometrics. 1988;31(4):499-500.

31. Elsaid MI, John T, Li Y, Koduru S, Ali SZ, Catalano C, et al. Health care utilization and economic burdens of hemochromatosis in the United States: a population-based claims study. J Manag Care Spec Pharm. 2019;25(12):1377-86.

32. American Hemochromatosis Society. Facts about hemochromatosis/iron overload. Prevention through genetic testing website https ://www.americanhs.org/faq.htm. Accessed 21 Sept 2018.

33. Balwani M, Sardh E, Ventura P, Peiró PA, Rees DC, Stölzel U, et al. Phase 3 trial of RNAi therapeutic givosiran for acute intermittent porphyria. N Engl J Med. 2020;382(24):2289-301.

34. Cortez M. Alnylam surges on FDA approval of $\$ 575,000$ drug for rare disease. Bloomberg 2019 [cited 2020 June, 10]. https:// www.bloomberg.com/news/articles/2019-11-20/alnylam-ultra -rare-disease-drug-comes-with-ultra-high-price-tag. Accessed 20 Nov 2019

35. Balwani M, Wang B, Anderson KE, Bloomer JR, Bissell DM, Bonkovsky HL, et al. Acute hepatic porphyrias: recommendations for evaluation and long-term management. Hepatology (Baltimore, MD). 2017;66(4):1314-22.

36. Anderson KE, Bloomer JR, Bonkovsky HL, Kushner JP, Pierach CA, Pimstone NR, et al. Recommendations for the diagnosis and treatment of the acute porphyrias. Ann Intern Med. 2005;142(6):439-50.

37. Asahina Y, Tsuchiya K, Tamaki N, Hirayama I, Tanaka T, Sato $\mathrm{M}$, et al. Effect of aging on risk for hepatocellular carcinoma in chronic hepatitis $\mathrm{C}$ virus infection. Hepatology (Baltimore, MD). 2010;52(2):518-27.

38. Mantovani LG, Strazzabosco M. Healthcare costs associated with hepatocellular carcinoma and the value of care. Hepatology (Baltimore, MD). 2013;58(4):1213-4.

39. Ergen EN, Seidler E, Parekh S, Parker SRS. Is non-alcoholic steatohepatitis a predisposing factor to porphyria cutanea tarda? Photodermatol Photoimmunol Photomed. 2013;29(2):106-8.

40. Perumpail BJ, Khan MA, Yoo ER, Cholankeril G, Kim D, Ahmed A. Clinical epidemiology and disease burden of nonalcoholic fatty liver disease. World J Gastroenterol. 2017;23(47):8263-76.

41. von und zu Fraunberg M, Pischik E, Udd L, Kauppinen R. Clinical and biochemical characteristics and genotype-phenotype correlation in 143 Finnish and Russian patients with acute intermittent porphyria. Medicine (Baltimore). 2005;84(1):35-47.

42. Cortez-Pinto H, Gouveia M, dos Santos PL, Costa J, Borges M, Vaz CA. The burden of disease and the cost of illness attributable to alcohol drinking - results of a national study. Alcohol Clin Exp Res. 2010;34(8):1442-9.

43. Mellinger JL, Shedden K, Winder GS, Tapper E, Adams M, Fontana RJ, et al. The high burden of alcoholic cirrhosis in privately insured persons in the United States. Hepatology. 2018;68(3):872-82.

44. Christiansen AL, Brock A, Bygum A, Rasmussen LM, Jepsen P. Increased mortality in patients with porphyria cutanea tarda-a nationwide cohort study. J Am Acad Dermatol. 2020;83(3):81723. https://doi.org/10.1016/j.jaad.2019.07.082. 